\title{
Signing enhances memory like performing actions
}

\author{
HUBERT D. ZIMMER and JOHANNES ENGELKAMP \\ Saarland University, Saarbrücken, Germany
}

\begin{abstract}
In three experiments, we investigated the influence of the overt performance of signs on memory. Deaf and hearing participants studied lists of action phrases (Experiment 1) or nouns (Experiment 2) under standard verbal instruction, under the instructions to sign the verbal phrase, to symbolically perform the denoted action, or to carry out a prototypical action corresponding to each noun. Higher recall and recognition performances were observed when actions were performed than in the verbal encoding condition, and signing was as effective for memory as was enactment. Thus, overt signing can induce an enactment effect. In contrast, Experiment 3 demonstrated that performing an unrelated action did not. A unique but unrelated action was not memory efficient.
\end{abstract}

In so-called subject-performed tasks (SPTs), participants are required to symbolically perform a series of minitasks complying with verbal commands, such as tear up the paper. Participants perform these actions either with real objects or with imaginary objects, and during testing, they are required either to recall or to recognize the verbal commands. A well-established finding is that this type of encoding, in comparison with verbal encoding, consistently enhances memory (for reviews, see Engelkamp, 1998; Nilsson, 2000; Zimmer \& Cohen, 2001). This substantial memory enhancement is generally attributed to traces of the additional processes that are necessary for one to perform these actions overtly (see Zimmer, 2001).

When using sign language, signers perform hand movements that are structurally similar to the symbolic movements executed in SPTs. Actually, some results suggest that the movement information of these gestures is part of the memory representation of signed items, as has been postulated for movements in SPT. For instance, the signers' short-term memory is impaired if the signs of the list items are similar in form (see Poizner, Bellugi, \& Tweney, 1981; Shund, 1982). Intrusion errors tend to preserve the basic hand arrangement of the item originally presented (Bellugi, Klima, \& Siple, 1975). In recognition, false alarm rates increase if distractors have signs that are structurally similar to the study items (see, e.g., Frumkin \& Anisfeld, 1977). In terms of motor components, signing may thus be comparable to the performing of actions. If this holds true, and if the motor act per se is the core element of the SPT effect (see Engelkamp,

This research was supported by DFG Grant En 124/13 and DAAD Grant ACLS 315. We thank Marc Marschark from the National Technical Institute of the Deaf (Rochester, NY) for his help in designing the experiments and his consultation regarding sign language, and also Volker Kneip for his help in running the experiments. Correspondence concerning this article should be addressed to H. D. Zimmer, Department of Psychology, Saarland University, P. O. Box 151150, D-66041 Saarbrücken, Germany (e-mail: huzimmer@mx.uni-saarland.de).
2001), signing may induce SPT-like effects and enhance memory. 1

Nevertheless, given certain other results, one might come to a different prediction. With oral language, it has been found that the overt performance of the verbal components, such as writing down or saying words aloud, does not enhance memory (Bahrick \& Boucher, 1968; Durso \& Johnson, 1980; Paivio \& Csapo, 1973). An exception is recognition memory, for which a small positive effect has been reported (Conway \& Gathercole, 1990). For proficient signers, enacting signs plays the same role as does generating phonological structures in oral languages, and therefore signing may behave as the overt production of oral language. In this case, signing should not have a considerable effect on memory.

\section{EXPERIMENT 1}

In Experiment 1, we compared memory for action phrases that were signed by deaf people during encoding with memory for action phrases that were studied in a verbal task (VT) by reading the phrases. Half of the deaf participants signed the items during study and during recall; the other half signed during study only and their recall was written. Finally, in a group composed of hearing persons, we contrasted a VT with a standard SPT condition for the same items. If overt movements are a critical component of the SPT effect, one should observe better memory for performed than for verbally encoded items with both types of study enactment; if performing the nonverbal referent of the phrase is critical instead, SPT but not signing should enhance memory.

\section{Method}

Participants. Forty-eight persons participated in the experiment. Thirty-two of them were deaf people who were recruited from a local informal organization for the deaf. All had been deaf since birth, and all reported themselves to be fluent in sign language and to use it as their primary language. Sixteen participants were hearing persons of the same age and educational level who mainly belonged to local sport clubs. All the participants studied one list 
under VT, as well as a second list while carrying out instructions (either signing or executing the action, respectively).

Material and Design. We constructed action phrases, each one consisting of a noun and a verb. To ensure that the items had unambiguous signs, the phrases were signed by a proficient signer in a prestudy. Only items were used for which a unique sign in German Sign Language exists.

The phrases were presented in 24-point letters on index cards. The 60 items were randomly assigned to one of two lists of 30 items each. One of the lists was presented under reading instructions, and the other one with either SPT or signing instructions. The orders of these encoding conditions, the order of the lists, and the assignment of lists to conditions were counterbalanced. For recognition, $60 \mathrm{ad}-$ ditional items were constructed to serve as distractors.

Procedure. The experiment was conducted by a hearing research assistant who knew sign language. The participants were given written instructions. They were required to read the items silently in the verbal condition, to perform the denoted action with a fictitious object in the SPT condition, and to sign the phrase in the signing condition. The items were presented for $3 \mathrm{sec}$ per item. After presentation, $3 \mathrm{~min}$ were given for recall. Half of the deaf participants were instructed to write the items down, and the other half were instructed to sign the items. In the latter case, the experimenter checked their correctness on line. A self-paced recognition test followed. The participants were presented a list showing old and new items in random order, and they marked each item as old or new. After that, the next study sequence started with the corresponding instruction.

\section{Results and Discussion}

The average proportion of recalled items is given in Table 1. We analyzed the free recall data in an analysis of variance (ANOVA) with the factors of encoding condition (reading, performing) and experimental group (hearing participants, deaf participants, and written recall; deaf participants and signed recall). As a third factor, we included the sequence of study conditions (performing first, reading first). The encoding condition $\left[F(1,42)=76.71, M S_{\mathrm{e}}=.003\right]$ and the group factor $\left.\mathrm{F}(2,42)=10.13, M S_{\mathrm{e}}=.013\right]$ were highly significant $(p<.001)$, whereas all other effects were not $(F<1)$. Free recall performance was generally about .10 higher after performing either signs or actions than after reading during encoding. For the deaf participants, the type of recall was unimportant (.18 verbal, .20 signed) but both groups recalled fewer items than did the hearing participants (.30).

The recognition data were calculated as discrimination scores defined as the proportion of hits minus the propor- tion of false alarms $\left(P_{\mathrm{R}}\right)$. In an ANOVA with the factors group and encoding condition of these $P_{\mathrm{R}}$ scores, the encoding effect was highly significant $[F(1,45)=191.45$, $\left.M S_{\mathrm{e}}=.008, p<.001\right]$. The discrimination score was .68 for the reading condition and .94 for the two performance conditions. The advantage of enactment was comparable for performing actions (.23) and signing (.28) $(F<1)$. Furthermore, recognition performances were somewhat lower for the deaf participants $(.78, .81)$ than for the hearing participants $(.85)$, as planned comparisons showed $\left[F(1,45)=4.56, M S_{\mathrm{e}}=.014, p<.05\right]$. Hit rates were higher after performing (.96) than after verbal encoding (.80), and false alarms were lower after performing (.03) than after verbal encoding (.12).

These data clearly show that signing can enhance memory. It made no difference whether actions or signs were performed during study. Both conditions showed higher memory performances than did verbal encoding. The null effect of the mode of recall shows that the writing capabilities of the deaf participants were not an issue here. Moreover, this replicates what has earlier been observed in SPT studies. Performing actions during recall is not memory efficient (see Kormi-Nouri \& Nilsson, 2001). Signing and performing seem to take effect during encoding. Encoding items by performing actions causes more efficient memory traces than does verbal encoding. How these traces are reactivated is less important (see Zimmer, 2001).

\section{EXPERIMENT 2}

Although the results of Experiment 1 were clear-cut, their validity might be reduced. It is sometimes discussed that signs are globally iconic; that is, their form resembles some aspect of what they denote (Frishberg, 1975). The phrases of Experiment 1 were selected because of the uniqueness of their sign equivalents, and their "iconicity" was not checked. One might therefore speculate that the memory effect of signing is in reality an SPT effect. A subset of items that was iconic might have activated their action-related movements and this might have caused the memory effect, just as in SPT. Two indirect arguments oppose this interpretation. First, both memory effects were numerically equivalent, which

Table 1

Means and Standard Errors of Proportion Recalled and of Recognition Scores $\boldsymbol{P}_{\mathrm{R}}$ (Proportion of Hits Minus Proportion of False Alarms) for the Three Groups of Subjects, Dependent on Encoding Condition in Experiment 1

\begin{tabular}{|c|c|c|c|c|c|c|c|c|}
\hline \multirow[b]{3}{*}{ Group } & \multicolumn{4}{|c|}{$\begin{array}{c}\text { Free Recall } \\
\text { Encoding at Study }\end{array}$} & \multicolumn{4}{|c|}{$\begin{array}{c}\text { Recognition } \\
\text { Encoding at Study }\end{array}$} \\
\hline & \multicolumn{2}{|c|}{ Reading } & \multicolumn{2}{|c|}{ Performing } & \multicolumn{2}{|c|}{ Reading } & \multicolumn{2}{|c|}{ Performing } \\
\hline & $M$ & $S E$ & $M$ & $S E$ & $M$ & $S E$ & $M$ & $S E$ \\
\hline Hearing, written recall & .24 & .02 & $.36^{*}$ & .03 & .74 & .03 & $.95^{*}$ & .02 \\
\hline Deaf, written recall & .13 & .02 & $.23 \dagger$ & .03 & .68 & .03 & $.94 \dagger$ & .02 \\
\hline Deaf, signed recall & .16 & .02 & $.24 \dagger$ & .03 & .64 & .03 & $.92 \dagger$ & .02 \\
\hline
\end{tabular}

*Performing means performing the denoted actions during study. †Performing means signing the phrases during study. 
should not have been the case if signing was efficient only with a subset of the list items, and second, the iconicity of signs had already been shown to be irrelevant for other memory tasks, such as short-term memory (Poizner et al., 1981). However, in spite of these indirect arguments, we wanted to have direct empirical results that would dispel the reinterpretation of the signing effect as a concealed action effect.

One way to check for iconicity is to select the phrases in such a way that the movement patterns of signs clearly differ from the denoted actions. Since German Sign Language is not well standardized, it was difficult to guarantee this for the numerous action phrases we needed. Yet unlike action phrases, many nouns have unique signs that have a motor pattern different from the one executed as one performs an action with the denoted object. In Experiment 2, we selected such nouns. We presented these nouns, and in the signing condition, we instructed participants either to sign the nouns or, in the SPT condition, to perform a prototypical action corresponding to the noun. In both conditions, overt movements were performed, but the movements of the signs and of the actions were clearly unrelated to each other. For comparison, in a third condition we asked the participants to read the nouns.

Performing actions to nouns is not the common SPT paradigm, but it yields SPT effects too (see, e.g., KormiNouri, Nyberg, \& Nilsson, 1994). We therefore expected results similar to those of Experiment 1: Nouns that were signed should be remembered better than verbally encoded nouns, and just as well as those in accordance with which prototypical actions were performed.

In Experiment 2, we also investigated whether the practice of sign language would be of any importance. For deaf participants, sign language is the primary language, whereas for hearing interpreters, signing is a secondary language. Deaf participants may produce signs "directly," whereas hearing participants "translate" the phonological code into the sign code. If this difference causes different "levels of processing," and if this is critical for the enactment effect, hearing participants might show a more pronounced effect of signing than that for deaf participants. On the other hand, the result that both groups show comparable effects would suggest that overt performance is the critical element and not additional processing.

\section{Method}

Participants. Twenty-four hearing participants who were interpreters or sign-language teachers took part in the experiment, and 24 young deaf people from the same population as that for the deaf participants in Experiment 1 took part.

Material, Design, and Procedure. We constructed three lists of 24 items each. All 72 items were nouns that denoted real objects. The participants studied all three lists-each under a different encoding instruction - with a free recall after each list. The lists were counterbalanced over conditions. In addition, we constructed a recognition list that included all the old items, and a further set of 72 new items. This list was used in a final recognition test.

Each participant underwent all three encoding conditions: reading, performing a prototypical action in accordance with the noun, and signing the word. The sequence in which these three conditions were realized was counterbalanced. Except for the difference in the encoding instruction, the procedure was the same as in Experiment 1.

\section{Results and Discussion}

The free recall performances and the corrected recognition scores $\left(P_{\mathrm{R}}\right.$ scores) are reported in Table 2 . All the data were analyzed in a $3 \times 2$ ANOVA with the factors of encoding condition and group. For free recall, the only significant effect was the type of encoding. Participants recalled less in the reading condition (.32) than in the SPT $(.44)$ and the signing $(.47)$ conditions $[F(2,92)=39.02$, $\left.M S_{\mathrm{e}}=.008, p<.001\right]$. The corrected recognition scores were the same in SPT (.88) and in the signing condition (.90), both of which were much better than the reading condition $(.56)\left[F(2,92)=161.36, M S_{\mathrm{e}}=.108, p<.001\right]$. The corresponding hit rates were $.94, .96$, and .62 respectively, with a false alarm rate of .07 . No other effect was significant, although there was a small but insignificant advantage for the deaf participants over the hearing participants in the reading condition $[F(1,46)=3.26$, $\left.M S_{\mathrm{e}}=.0245, p<.08\right]$.

These results exactly replicated the results of Experiment 1 with a completely different set of items that had signs with a different movement pattern than the one executed while participants were performing typical actions with the denoted objects. Signing the nouns strongly enhanced memory and it did so to the same extent as did performing typical actions with the denoted objects. Still, one could doubt that we had been successful in manipulating the iconicity of signs. To check this, we asked two native signers to assess, on a scale from 1 (dissimilar) to 5 (similar), whether the sign was iconic to the denoted action; that is, whether the motor pattern of the sign and the action were similar. As suspected, the movements of signs and actions in Experiment 1 were quite similar (4.5 on the average), whereas in Experiment 2, they were different (1.6). To check whether the iconicity influenced memory, we selected, from Experiment 1, only the items that were very similar $(\geq 4, n=48)$, and from Experiment 2 , those that were clearly dissimilar $(\leq 2, n=61)$, and we analyzed the free recall data from both experiments within one ANOVA with the factors of iconicity and en-

Table 2

Means and Standard Errors of Proportion Recalled and

$P_{\mathrm{R}}$ Scores, Dependent on Encoding Condition and Group in Experiment 2

\begin{tabular}{|c|c|c|c|c|c|c|}
\hline \multirow[b]{3}{*}{ Group } & \multicolumn{6}{|c|}{ Encoding Condition } \\
\hline & \multicolumn{2}{|c|}{ Reading } & \multicolumn{2}{|c|}{ SPT } & \multicolumn{2}{|c|}{ Signing } \\
\hline & $M$ & $S E$ & $M$ & $S E$ & $M$ & $S E$ \\
\hline \multicolumn{7}{|c|}{ Free Recall } \\
\hline Hearing & .34 & .02 & .45 & .02 & .48 & .02 \\
\hline Deaf & .30 & .02 & .43 & .02 & .46 & .02 \\
\hline \multicolumn{7}{|c|}{ Recognition } \\
\hline Hearing & .52 & .03 & .87 & .02 & .90 & .02 \\
\hline Deaf & .60 & .03 & .88 & .02 & .90 & .02 \\
\hline
\end{tabular}


coding condition. Two significant main effects were found. Signing (Experiment 1, .24; Experiment 2, .46) caused better memory performance than did verbal encoding (Experiment $1, .17$; Experiment 2, .31) $\left[F(1,54)=56.74, M S_{\mathrm{e}}=\right.$ $.007, p<.001]$, and nouns were recalled better than phrases $\left[F(1,54)=88.62, M S_{\mathrm{e}}=.011, p<.001\right]$. In addition, the interaction was significant $\left[F(1,54)=5.94, M S_{\mathrm{e}}=.007\right.$, $p<.05]$. Although nouns were less iconic than phrases, numerically they had a stronger signing effect (.15) than did phrases (.08). Because the effect of performing was observed in both experiments, we concluded that iconicity was not relevant for the memory enhancement by signing.

On the basis of these results, we assume that both types of actions enhance memory by means of the same principle. Signs and actions share the characteristic that their item-specific movement information is (preexperimentally) associated with the verbal component. If these components get part of the memory trace either by signing the items or by performing the actions, item-specific information is enhanced and this promotes remembering the item.

\section{EXPERIMENT 3}

The latter assumption implies that an action, which is performed in accordance with an item, enhances memory only if it is related to the item. Thus, the effects of enactment as well as of signing are not only a consequence of an unspecific arousal caused by the overt activity, but also of the activation of item-specific movement components-be it a sign or a meaningful action. Performing an action that is unrelated to the item should not be memory effective even though it is an overt performance and it is unique for the studied item. In Experiment 3 , we tested this prediction.

A noun was followed soon afterwards by a verb that denoted either an action that was related to the noun or an action that was not. For instance, the noun stone could be followed either by the verb throw (related) or by the verb drink (unrelated). Participants were instructed to memorize the noun and to perform the action denoted by the verb without making an attempt to combine both components. If the relatedness of the movement is relevant for integrating an item into memory, the unrelated movement should cause worse memory than the related one; on the other hand, if the effect is an (unspecific) activation effect, both types of encoding should lead to good memory performances.

\section{Method}

Participants. Twenty-four hearing students took part in the experiment for payment. Each studied one list of nouns with relevant actions and one with irrelevant actions.

Material, Design, and Procedure. For each noun from Experiment 2, two verbs were generated, one representing a meaningful action regarding the noun, and one unrelated to the noun. In the latter case, we used verbs that denoted an action that could not be sensibly performed with the object. Lists of 24 actions were con- structed, and the participants studied one of the lists with the relevant and one with the irrelevant action verb. Nouns were counterbalanced over these conditions. Each list was followed by a written recall of the nouns. During study, the nouns were presented one by one on a computer monitor. First, the noun was presented for $2 \mathrm{sec}$ on its own, and then the verb appeared. For $1 \mathrm{sec}$, both words remained visible; then the noun vanished and the verb remained for another second. When the verb was presented, the denoted action was to be performed. After an interstimulus interval of $500 \mathrm{msec}$, the next trial started.

\section{Results and Discussion}

Participants recalled more nouns with related actions $(.51, S D=.12)$ than if an irrelevant action was performed $(.28, S D=.13)$. The former was in the same range as the enactment conditions of Experiment 2 (.43-.48); the latter was similar to the results for corresponding reading condition (.30-.34). The difference between these two conditions was highly reliable $[t(24)=8.73, p<.001]$. This result demonstrates that performing an action while encoding an item does not generally enhance memory. It does so only if the action is related to the item to be remembered. This effect gives an idea of why signing is memory efficient, too. For a proficient signer, the sign is an overt action that is associated with the item just as the denoted action is associated with the item for a hearing participant.

\section{GENERAL DISCUSSION}

The data from the first two experiments demonstrate that signing has a clear positive effect on memory and that this effect is as strong as the effect of performing the actions (SPT effect). These effects were observed in recall as well as in recognition; they were larger for recognition than for recall; and they were discovered for action phrases as well as for nouns. Although the recognition performances might have been influenced by the preceding free recall, the hit rates were so high (about .95) that we can nevertheless conclude that there was a contribution of performing to recognition that was independent of recall.

Signing obviously caused the same memory advantage as did performing the action, and for the memory effect, it was not important whether nouns or action phrases were signed. Independently of us, von Essen and Nilsson (2003) conducted a similar experiment. Besides verbal encoding, they used two additional control conditions. Their participants imagined performing the action or imagined the actions and in addition translated the Swedish phrases into English. Signing was as good as performing, and both were better than the remaining tasks. The fact that translation was not as efficient as signing demonstrates that the signing effect is not only a semantic elaboration effect caused by translating the verbal code into sign language. This is also supported by our observation that the signing effect was as large for deaf as for hearing participants (Experiment 2), if one considers that for hearing participants the verbal lan- 
guage and for deaf participants the sign language is the dominant one. Overall, these experiments substantiate the hypothesis that it is the overt performance that causes the memory effect of enactment; and that this effect is independent of the type of action that was performedthat is, a unique action or a sign. As Experiment 3 showed, the memory advantage disappeared if the performed action was unrelated to the item.

On the basis of these and related results (see Zimmer, 2001), we believe that it is sufficient for the generation of an enactment effect that in addition to conceptual processing an action component is activated during encoding that is unique for and associated with the item to be remembered. We assume that action-specific information is encoded when the movement is overtly performed, and that this information is integrated into the memory record if the action is preexperimentally associated with the item. During recall or recognition, this additional information enhances the recollection of the item. Signs as well as meaningful actions performed in accordance with words have this capability.

Before closing, a remark seems to be advisable. We do not postulate that any form of overt signing has this positive effect on memory. There may be encoding conditions in which the overt performance of signs does not enhance memory in comparison with a "silent" encoding of the signs. For example, we do not know what would happen if the to-be-performed signs were taught to participants by their watching an experimenter who was signing. We know from SPT research that an "imitating" condition causes less pronounced enactment effects than does performing actions in response to verbal commands (Zimmer \& Engelkamp, 1996). A similar result is likely for signing. Further experiments must show how different input conditions modify the effect of overt performance of signs on memory. Our experiments have shown that action information provided by signing can have the same memory effect as does performing the denoted action, and this is what we wanted to show.

\section{REFERENCES}

BAhrick, H. P., \& Boucher, B. (1968). Retention of visual and verbal codes of the same stimuli. Journal of Experimental Psychology, 78, 417-422.

Bellugi, U., Klima, E. S., \& Siple, P. (1975). Remembering in signs. Cognition, 3, 93-135.

Conway, M. A., \& Gathercole, S. E. (1990). Writing and long-term memory: Evidence for a "translation" hypothesis. Quarterly Journal of Experimental Psychology, 42A, 513-527.

Durso, F. T., \& Johnson, M. K. (1980). The effects of orienting tasks on recognition, recall and modality confusion of pictures and words. Journal of Verbal Learning \& Verbal Behavior, 19, 416-429.

EngelKamp, J. (1998). Memory for actions. Hove, U.K.: Psychology Press.

EngelKamp, J. (2001). Action memory: A system-oriented approach. In H. D. Zimmer, R. L. Cohen, M. J. Guynn, J. Engelkamp, R. KormiNouri, \&. M. A. Foley (Eds.), Memory for action: A distinct form of episodic memory? (pp. 49-96). New York: Oxford University Press.

FrISHBERG, N. (1975). Arbitrariness and iconicity: Historical change in American Sign Language. Language, 51, 696-719.

Frumkin, B., \& Anisfeld, M. (1977). Semantic and surface codes in the memory of deaf children. Cognitive Psychology, 9, 475-493. Kormi-Nouri, R, \& NiLsson, L.-G. (2001). The motor component is not crucial! In H. D. Zimmer, R. L. Cohen, M. J. Guynn, J. Engelkamp, R. Kormi-Nouri, \& M. A. Foley (Eds.), Memory for action: A distinct form of episodic memory? (pp. 97-111). New York: Oxford University Press.

Kormi-Nouri, R., Nyberg, L., \& Nilsson, L.-G. (1994). The effect of retrieval enactment on recall of subject-performed tasks and verbal tasks. Memory \& Cognition, 22, 723-728.

NiLsson, L.-G. (2000). Remembering actions and words. In F. I. M. Craik \& E. Tulving (Eds.), The Oxford handbook of memory (pp. 137-148). Oxford: Oxford University Press.

Paivio, A., \& Csapo, K. (1973). Picture superiority in free recall: Imagery or dual coding? Cognitive Psychology, 5, 176-206.

Poizner, H., Bellugi, U., \& Tweney, R. D. (1981). Processing of formational, semantic, and iconic information in American Sign Language. Journal of Experimental Psychology: Human Perception \& Performance, 7, 1146-1159.

Shund, M. A. (1982). Sign-based short-term coding of American Sign Language signs and printed English words by congenitally deaf signers. Cognitive Psychology, 14, 1-12.

von Essen, J. D., \& NiLsson, L.-G. (2003) Memory effects of motor activation in subject-performed tasks and sign language. Psychonomic Bulletin \& Review, 10, 445-449.

Zimmer, H. D. (2001). Why do actions speak louder than words: Action memory as a variant of encoding manipulations or the result of a specific memory system? In H. D. Zimmer, R. L. Cohen, M. J. Guynn, J. Engelkamp, R. Kormi-Nouri, \&. M. A. Foley (Eds.), Memory for action: A distinct form of episodic memory? (pp. 151-198). New York: Oxford University Press.

Zimmer, H. D., \& Cohen R. L. (2001). Remembering actions: A specific type of memory? In H. D. Zimmer, R. L. Cohen, M. J. Guynn, J. Engelkamp, R. Kormi-Nouri, \&. M. A. Foley (Eds.), Memory for action: A distinct form of episodic memory? (pp. 3-24). New York: Oxford University Press.

Zimmer, H. D., \& Engelkamp, J. (1996). Routes to actions and their efficacy for remembering. Memory, 4, 59-78.

\section{NOTE}

1. When we submitted this paper we heard that independently of us von Essen and Nilsson had submitted a manuscript in which they tested the memory-efficiency of signing as we did in the following experiment. That study is included in the present issue of Psychonomic Bulletin \& Review on pp. 445-449.

(Manuscript received April 3, 2001; revision accepted for publication April 1,2003.) 